\title{
BMJ Open Protocol for hospital based-surveillance of cerebral palsy (CP) in Hanoi using the Paediatric Active Enhanced Disease Surveillance mechanism (PAEDS- Vietnam): a study towards developing hospital-based disease surveillance in Vietnam
}

\author{
Gulam Khandaker, ${ }^{1,2}$ Nguyen Van Bang, ${ }^{3}$ Trịnh Quang Dũng, ${ }^{4}$ \\ Nguyen Thi Huong Giang, ${ }_{4}^{4}$ Cao Minh Chau, ${ }^{5}$ Nguyen Thi Van Anh, ${ }^{6}$ \\ Nguyen Van Thuong, ${ }^{7}$ Nadia Badawi, ${ }^{8,9}$ Elizabeth $\mathrm{J} \mathrm{Elliott}{ }^{10,12}$
}

To cite: Khandaker G, Van Bang N, Dũng TQ, et al. Protocol for hospital based-surveillance of cerebral palsy (CP) in Hanoi using the Paediatric Active Enhanced Disease Surveillance mechanism (PAEDS-Vietnam): a study towards developing hospitalbased disease surveillance in Vietnam. BMJ Open 2017;0:e017742. doi:10.1136/ bmjopen-2017-017742

- Prepublication history and additional material for this paper are available online. To view please visit the journal (http:// dx.doi.org/10.1136/bmjopen2017-017742)

Received 22 May 2017 Revised 22 August 2017 Accepted 8 September 2017

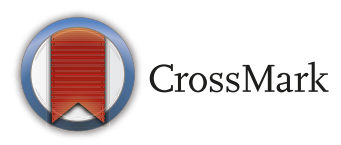

For numbered affiliations see end of article.

\section{Correspondence to}

Dr Gulam Khandaker; gulam.khandaker@health.nsw. gov.au

\section{ABSTRACT}

Introduction The epidemiology, pathogenesis, management and outcomes of cerebral palsy (CP) in lowincome and middle-income countries including Vietnam are unknown because of the lack of mechanisms for standardised collection of data. In this paper, we outline the protocol for developing a hospital-based surveillance system modelled on the Paediatric Active Enhanced Disease Surveillance (PAEDS) system in Australia. Using PAEDS-Vietnam we will define the aetiology, motor function and its severity, associated impairments, and nutritional and rehabilitation status of children with $\mathrm{CP}$ in Hanoi, Vietnam. These essential baseline data will inform future health service planning, health professional education and training, and family support.

Methods and analysis This is a hospital-based prospective surveillance of children with CP presenting to the rehabilitation, neurology and general paediatric services at the National Children's Hospital and St Paul Hospital in Hanoi. We will use active, prospective daily case-finding for all children with $\mathrm{CP}$ aged $<18$ years who are hospitalised or present to outpatient departments. Following parental consent, data will be collected using a modified version of the Australian Cerebral Palsy Register questionnaire. The data collection form has been developed in consultation with local and international experts and translated into Vietnamese. Information collected will include demographics, maternal health and birth history, type and severity of $\mathrm{CP}$, known risk factors for $\mathrm{CP}$, and nutrition, immunisation, education and rehabilitation status.

Ethics and dissemination This study was approved by the Hanoi Medical University Institutional Review Board (decision no 1722) and The University of Sydney Human Research Ethics Committee (approval no 2016/456). Establishment of PAEDS-Vietnam will enable hospitalbased surveillance of CP for the first time in Vietnam. It will identify preventable causes of CP, patient needs and service gaps, and facilitate early diagnosis and intervention. Study findings will be disseminated through local and international conferences and peer-reviewed publications.

\section{INTRODUCTION}

Cerebral palsy (CP) is the major global cause of childhood disability. Based on data from high-income countries, it affects up to 17 million people worldwide, with a prevalence of approximately 2 per 1000 live births. ${ }^{1}$ The prevalence of $\mathrm{CP}$ in low-income and middle-income countries is believed to be four to six times higher; however, few reliable data are available. ${ }^{23}$ In Vietnam it is estimated that 500000 people live with $\mathrm{CP}$ and that CP comprises 30\%-40\% of all childhood disability. ${ }^{45}$

The prevalence of CP likely varies between provinces in Vietnam. For example, CP prevalence was 0.6 per 1000 in the general population in Khanh Hoa and 1.5 per 1000 in the general population in Hatay. ${ }^{6}$ These data are limited by methodological quality of studies, including small sample sizes, and likely underestimates of CP prevalence. In one treatment centre in Vietnam, an increase in the number of cases with CP has been documented over time. In 1998 at that centre 394 (25.7\%) of all children admitted had CP. This increased by more than three times to $912(47.3 \%)$ of all admissions in 2002. ${ }^{6}$ In Vietnam, the proportion of children with $\mathrm{CP}$ who receive rehabilitation services is estimated at between $30 \%$ 
and $74 \%$; however, this cannot be accurately determined without good epidemiological data. ${ }^{6}$

The Vietnamese Ministry of Health has recently recognised service delivery for $\mathrm{CP}$ as a public health priority. ${ }^{4}$ Furthermore, a recent health reform in Vietnam has established universal health coverage that will provide free-of-charge treatment for children with $\mathrm{CP}$ who are aged 6 years or less. Children over 6 years of age who attend school are only covered by voluntary health insurance, requiring guardians to purchase treatment based on their ability to pay. ${ }^{7}$ There are no Vietnamese data indicating the age of diagnosis of $\mathrm{CP}$ nor the proportion of children who attend mainstream school. In a recent study from Bangladesh, we found that the mean age of diagnosis of CP was 4.9 years and only $16 \%$ of children with CP attended mainstream school, the remainder having no access to education (preliminary results from the Bangladesh Cerebral Palsy Register study). ${ }^{8}$

Reported treatment of CP in Vietnam is commonly a combination of traditional and modern medicine. Traditional medicine includes massage, reflexology, use of electrical stimulation or water magnets, and stimulated ventilation with an electric current. ${ }^{5}$ A holistic treatment approach, provided following establishment of a centre for social assistance for disadvantaged children, was effective in improving quality of movement through physiotherapy in $92 \%$ of children and providing special/ inclusive education. ${ }^{9}$

In Vietnam there is an urgent need to understand the burden of CP, patient needs and service gaps. However there is no disease surveillance, there are no universally accepted treatment guidelines, and there is no information on the uptake of evidence-based diagnostics or treatments and policy planning. The lack of rigorous epidemiological data limits capacity to plan for future disability services.

We aim to develop a hospital-based surveillance system modelled on the Paediatric Active Enhanced Disease Surveillance (PAEDS) system operating in Australia. Using PAEDS-Vietnam we will define the aetiology, motor function, severity, associated impairments, and nutritional and rehabilitation status of children with CP presenting to two hospitals in Hanoi, Vietnam. Collection of these essential baseline data will inform future health service planning, and need for health professional training and family support. Our experience will establish the feasibility of extending the surveillance system for use in other conditions and potentially to the national level.

\section{METHODS AND ANALYSIS}

\section{Aims and objectives}

The overall aims of this study are to (1) implement a hospital-based surveillance system to identify children presenting with CP in Hanoi that is modelled on PAEDS; (2) collect baseline information on the known risk factors, clinical presentation (motor function/severity, associated impairments, nutritional and rehabilitation status), service use and needs of children with $\mathrm{CP}$ in Hanoi, Vietnam; and (3) assess the feasibility of a national hospital-based paediatric disease surveillance mechanism for Vietnam.

Our specific objectives are to identify and characterise children with CP presenting to the National Children's Hospital (NCH) and St Paul Hospital in Hanoi, Vietnam in order to:

1. document the burden of CP in these two hospitals and estimate the prevalence of CP in Hanoi province

2. define the aetiology of CP

3. document the motor impairment and severity of $\mathrm{CP}$ using the Gross Motor Function Classification System (GMFCS) and the Manual Ability Classification System

4. describe associated impairments in children with $\mathrm{CP}$

5. assess the nutritional status of children with $\mathrm{CP}$

6. assess the rehabilitation status of children with $\mathrm{CP}$

7. evaluate the strengths and limitations of a hospitalbased disease surveillance mechanism.

\section{Overview of study design}

This is a hospital-based surveillance study to identify a prospective cohort of children with $\mathrm{CP}$ who attend the rehabilitation, neurology and general paediatric services (inpatient and outpatient) at the NCH and St Paul Hospital in Hanoi, Vietnam.

\section{Description of the participating hospitals}

$\mathrm{NCH}$ is a 1200-bed tertiary paediatric hospital that provides services for nearly 40000 inpatients and 350000 outpatients each year from northern Vietnam. St Paul Hospital is a general medical facility with 150 paediatric beds. Both hospitals are located in central Hanoi, the capital of Vietnam.

\section{Surveillance methods/activities}

We will establish a method for active, hospital-based surveillance to identify children with $\mathrm{CP}$ attending the two hospitals (ie, PAEDS-Vietnam). This system will be based on the highly successful, government-funded PAEDS system in Australian, established by the Australian Paediatric Surveillance Unit in collaboration with the National Centre for Immunisation Research and Surveillance of Vaccine Preventable Diseases in 2007. ${ }^{10}$ PAEDS is currently active in five Australian states and collects data on six conditions (ie, acute flaccid paralysis, intussusception, varicella and herpes zoster, pertussis, febrile seizures, and acute childhood encephalitis). Clinicians are appointed specifically to identify new admissions or presentations with the condition of interest on a daily basis. This is achieved by daily contact with key collaborators (eg, directors of the rehabilitation ward) and review of hospital intake data (eg, ward admissions). This process allows for the timely collection of clinical and laboratory data on hospitalised children and has proven invaluable for monitoring selected conditions of national interest (eg, varicella requiring hospitalisation $)^{11}$ and responding 
to epidemiological emergencies (eg, the 2009 influenza pandemic).$^{1213}$

\section{Study participants}

Participants of the study are all children aged less than 18 years with $\mathrm{CP}$ attending as an inpatient or outpatient to one of the participating hospitals during the study period.

\section{Case definition}

We have adopted the approach used by Surveillance of Cerebral Palsy in Europe and the Australian Cerebral Palsy Register, ${ }^{14} 15$ which allows use of any definition that includes the following five key elements. ${ }^{16-18}$

Cerebral palsy:

1. is an umbrella term for a group of disorders

2. is a condition that is permanent but not unchanging

3. involves a disorder of movement and/or posture and of motor function

4. is due to a non-progressive interference, lesion or abnormality

5. the interference, lesion or abnormality originates in the immature brain.

\section{Inclusion/exclusion criteria}

A 'case' must fulfil the criteria contained in the five definitional elements above. In children aged $<5$ years, when a diagnosis of ' $\mathrm{CP}$ ' is made, the diagnosis must be confirmed when the child reaches 5 years of age. Most children will be followed clinically in the hospital setting until therapy is established. Only those children aged less than 5 years will be specifically followed up by the investigator team to confirm the diagnosis once they are over the age of 5 . If new information becomes available for a child participating in the study, their entry may be updated, which may involve inclusion or exclusion. ${ }^{15}$

Children with intellectual disability of a known cause, neuromuscular disorders, genetic disorders (eg, trisomy 21, tuberous sclerosis), a known epilepsy syndrome, progressive neurodegenerative disorders, brain malignancy or traumatic brain injuries will be excluded from this study.

\section{Consent}

Written consent for study participation will be obtained by Vietnamese surveillance medical officers (ie, third party) using participation information and consent forms written in Vietnamese. The treating physician will then complete the data collection form. The surveillance medical officers will not have any role in patients' clinical care; thus, coercion is unlikely and participation will not influence clinical care.

Children with CP aged less than 18 years will be recruited in this study and parental consent alone will be sought for two reasons:

1. Children with $\mathrm{CP}$ often have intellectual/cognitive impairment and their ability to give informed consent is variable and uncertain.

2. Children aged less than 18 years are considered minors in Vietnam, requiring their parents/primary caregivers to take full responsibility/authority for any decisions related to their medical care and participation in research.

However, children aged over 14 years and with an appropriate comprehension level will be asked for assent. To consider a child for assent, the study investigators will take into account the child's age, maturity and psychological state to determine whether the child is capable of giving a meaningful assent.

\section{Case ascertainment}

Before commencing surveillance, we will provide, and train local clinicians to use, internationally recognised diagnostic guidelines to make the diagnosis of $\mathrm{CP}^{19}{ }^{19} \mathrm{Clini}-$ cians at each site will identify eligible children on a daily basis (active case ascertainment). Clinical data relevant to the study protocol will be recorded on a data collection form. We will train medical officers (paediatric doctors or trainees) to identify and characterise cases in each surveillance hospital for the duration of the study. Local study physicians (eg, general paediatricians, rehabilitation paediatricians, paediatric neurologists) will make the diagnosis of $\mathrm{CP}$ or confirm the diagnosis on referred cases. They will be trained in recently published CP diagnostic algorithm. ${ }^{19}$

The medical officers will be responsible for recruiting participants from different hospital departments (eg, rehabilitation, neurology, general paediatrics and outpatient clinics) and will be supervised by the collaborating lead investigators from the surveillance hospitals, who will verify the type and severity of $\mathrm{CP}$ recorded. Clinical data on all cases identified will be reviewed by the investigator group for potential misdiagnosis. In contentious cases the opinion of a paediatric neurologist will be sought. Data quality and completeness will be checked regularly by the investigators and senior clinicians at both hospitals. Table 1 shows the components of the proposed surveillance mechanism to be used in PAEDS-Vietnam.

Table 1 Components of Paediatric Active Enhanced Disease Surveillance-Vietnam surveillance methods

\begin{tabular}{|c|c|}
\hline $\begin{array}{l}\text { Surveillance } \\
\text { hospitals }\end{array}$ & $\begin{array}{l}\text { National Children's Hospital and St Paul } \\
\text { Hospital }\end{array}$ \\
\hline $\begin{array}{l}\text { Surveillance } \\
\text { departments }\end{array}$ & $\begin{array}{l}\text { Rehabilitation, neurology, general paediatric } \\
\text { outpatient }\end{array}$ \\
\hline $\begin{array}{l}\text { Case } \\
\text { ascertainment }\end{array}$ & $\begin{array}{l}\text { Daily review of both inpatient and } \\
\text { outpatients list }\end{array}$ \\
\hline $\begin{array}{l}\text { Consent and } \\
\text { recruitment }\end{array}$ & $\begin{array}{l}\text { Participating study physicians } \\
\text { Defined inclusion/exclusion criteria }\end{array}$ \\
\hline $\begin{array}{l}\text { Data quality } \\
\text { monitoring }\end{array}$ & $\begin{array}{l}\text { Named investigators at the participating } \\
\text { institutes/departments }\end{array}$ \\
\hline Data entry & $\begin{array}{l}\text { Research officers/investigators entering } \\
\text { data to electronic data repository }\end{array}$ \\
\hline $\begin{array}{l}\text { Ongoing } \\
\text { monitoring }\end{array}$ & $\begin{array}{l}\text { Monthly study implementation meeting: all } \\
\text { investigators }\end{array}$ \\
\hline
\end{tabular}




\section{Data collection and quality assurance}

Standard data collection forms have been developed (see online supplementary appendix A) in consultation with international experts, translated into Vietnamese, and modified following review by Vietnamese investigators to ensure they are appropriate for the local setting. Data will be collected on demographics and primary health indicators, birth details of the child with CP and maternal pregnancy details, neurological and motor classifications of CP (ie, GMFCS), associated impairments, timing of CP, and immunisation, nutrition, education and rehabilitation status. Once the record form has been completed and checked by a research physician, data will be entered into the PAEDS-Vietnam electronic data repository. Cases will be de-identified and only the study investigators and nominated delegates will have access to identifiable data. The PAEDS-Vietnam secretariat and data coordinating centre will be located at Hanoi Medical University, Hanoi, Vietnam. Internal checks for data quality and data entry errors will be performed routinely in the data coordinating centre. The completeness of ascertainment will be checked by audit of hospital records and using capture-recapture methods. De-identified data will be shared regularly with the Sydney investigators using a secure portal.

\section{Statistical methods}

Descriptive epidemiological measures, such as rate of hospital presentations and prevalence of $\mathrm{CP}$, will be estimated from the surveillance data. Frequencies of different types of $\mathrm{CP}$ will be presented as a percentage with $95 \%$ CI. An estimate of prevalence of CP in Hanoi province will be calculated per 1000 child population with $95 \%$ CI. We will estimate the prevalence based on the hospital catchment population and proportion of children with CP attending those hospital for services. This method has previously been used successfully by our group to estimate the incidence of congenital rubella syndrome in Hanoi province. ${ }^{20}$ We will document the children's address (eg, district, province) to enable identification and of children coming from outside the Hanoi region and their exclusion from estimates of prevalence.

We will perform one-sample binomial tests (including t-tests) to compare proportions for demographic variables in children with $\mathrm{CP}$ and the most recent national census and demographic and health survey data to calculate the denominator population and enable comparisons. The Statistical Package for Social Sciences (IBM SPSS V.23) will be used for data handling and analysis.

\section{Confidentiality and privacy}

Coded, non-identifiable data will be stored on the PAEDS-Vietnam electronic database. The data set will be accessible by the administrator only, with computers protected by a secure password log-on instigated after 5 min of computer inactivity. No data will be stored on any researcher's personal or hospital computer. Only group data will be presented in reports or publications and no identifiable information will be made available or apparent through provision of specific personal or health characteristics. A non-identifiable data set will be shared with the lead investigators in Australia for data quality assessment and advanced statistical analysis.

\section{ETHICS AND DISSEMINATION \\ Study duration}

The pilot phase of the study will be for 6 months. During this period we will train the study investigators and participating physicians and develop the study implementation tools. Moreover, during the pilot phase we will gain a better understanding of the case load (ie, number of children with CP seeking medical care at participating hospitals). After the pilot phase we will conduct an interim evaluation of the surveillance mechanism. Once the pilot phase is successfully implemented, surveillance will be continued for another 18 months. The PAEDS-Vietnam mechanism will remain in place for potential use in other conditions.

\section{Study limitations}

Hospital-based surveillance of children with $\mathrm{CP}$ will likely overestimate children with severe CP or with comorbidities such as epilepsy as these children more often require hospitalisation. Moreover, we would not be able to precisely estimate the prevalence of $\mathrm{CP}$ as it is unlikely that all the children with $\mathrm{CP}$ will seek medical care in the participating hospitals.

\section{Dissemination}

This study will have a number of immediate social and public health benefits: (1) By identifying children with CP from participating hospitals, we will increase clinician knowledge and skills and facilitate early diagnosis and intervention. (2) This prospective surveillance will provide unique baseline data on the estimated prevalence and profile of children with CP and the aetiology and risk factors for CP in Hanoi, Vietnam. (3) This cohort could be used as a sampling frame for future research, for example, to intervention trials to evaluate cost-effective treatment strategies to promote functional abilities and limiting secondary impairments in children with CP. (4) PAEDS-Vietnam will establish the feasibility and utility of a hospital-based, prospective, active disease surveillance mechanism in Vietnam, including its strengths and limitations. Once established, this surveillance could be adapted to monitor any disease of public health importance and extended to have a nation reach.

\section{Author affiliations}

${ }^{1}$ Discipline of Child and Adolescent Health, The Children's Hospital at Westmead Clinical School, Sydney Medical School, The University of Sydney, Sydney, New South Wales, Australia

${ }^{2}$ Asian Institute of Disability and Development (AIDD), University of South Asia, Dhaka, Bangladesh

${ }^{3}$ Department of Paediatrics, Hanoi Medical University, Hanoi, Vietnam ${ }^{4}$ Rehabilitation Department, National Children's Hospital, Hanoi, Vietnam ${ }^{5}$ Rehabilitation Department, National Children's Hospital (Bach Mai Hospital), Hanoi, Vietnam 
${ }^{6}$ Medical Education and Skills-Lab, Hanoi Medical University, Hanoi, Vietnam

${ }^{7}$ Paediatrics Department, St Paul Hanoi Municipal Hospital, Hanoi, Vietnam

${ }^{8}$ Grace Centre for Newborn Care, The Children's Hospital at Westmead, Sydney

Medical School, The University of Sydney, Westmead, New South Wales, Australia

${ }^{9}$ Cerebral Palsy Alliance Research Institute, The University of Sydney, Sydney, New South Wales, Australia

${ }^{10}$ Discipline of Child and Adolescent Health, The Children's Hospital at Westmead Clinical School, Sydney Medical School, The University of Sydney, Sydney, New

South Wales, Australia

${ }^{12}$ Australian Paediatric Surveillance Unit, Kids Research Institute, Westmead, New South Wales, Australia

Acknowledgements We acknowledge the support of study coordinating and implementing partners from NCH and St Paul Hospital.

Contributors EJE together with GK and NVB conceptualised, designed and established this research study. EJE, GK and NVB also contributed to study design and were responsible for the development of the study materials. NVB developed the Vietnamese version of the data collection sheet. EJE, GK and NVB were responsible for ethics applications and reporting. NVB, TQD, NTHG, CMC, NTVA and NVT will be responsible for recruitment, data collection and overall conduct of the study in Vietnam. EJE, GK, NB, NVB and CMC will be providing specialist advice in this study. EJE and GK drafted the manuscript with input from all the coauthors. All authors have agreed the final version of the manuscript and were involved in the decision to submit the manuscript. All authors read and approved the final manuscript.

Funding This study is funded by the Research Foundation of Cerebral Palsy Alliance (PG 6115). GK is supported by an NHMRC Health Early Career Fellowship (1054414). EJE is also supported by an NHMRC Practitioner Fellowship (457084 (MD1)).

Competing interests None declared.

Ethics approval University of Sydney Human Research Ethics Committee (approval no 2016/456) and Hanoi Medical University Institutional Review Board (HMU IRB decision no 1722).

Provenance and peer review Not commissioned; externally peer reviewed.

Open Access This is an Open Access article distributed in accordance with the Creative Commons Attribution Non Commercial (CC BY-NC 4.0) license, which permits others to distribute, remix, adapt, build upon this work non-commercially, and license their derivative works on different terms, provided the original work is properly cited and the use is non-commercial. See: http://creativecommons.org/ licenses/by-nc/4.0/

(C) Article author(s) (or their employer(s) unless otherwise stated in the text of the article) 2017. All rights reserved. No commercial use is permitted unless otherwise expressly granted.

\section{REFERENCES}

1. Ozmen M, Calişkan M, Apak S, et al. 8-year clinical experience in cerebral palsy. J Trop Pediatr 1993;39:52-4.

2. Cruz M, Jenkins R, Silberberg D. The burden of brain disorders. Science 2006;312:53:53b.
3. Donald KA, Samia P, Kakooza-Mwesige A, et al. Pediatric cerebral palsy in Africa: a systematic review. Semin Pediatr Neurol 2014;21:30-5.

4. Nhà xuất bản Y hoc. Tài liẹu số 10: Phuc hồi chúc năng cho trẻ bạ não. Hà Nội, Việt Nam: Nhà xuất bản Y học, 2008. 46 p.

5. CÔNG TY CP TM DV CSSK VĨNH ĐỨC. Phục hồi chức năng trẻ bại não. http://vinhduc.net.vn/kh\%C3\%A1m-v\%C3\%A0-\%C4\%91i\% E1\%BB\%81utr\%E1\%BB\%8B-vitl-tr\%E1\%BA\%BB-em/6151-ph\% E1\%BB\%A5c-h\%E1\%BB\%93ich\%E1\%BB\%A9c-n\%C4\%83ngtr\%E1\%BA\%BB-b\%E1\%BA\%A1i-n\%C3\%A3o

6. Nguyen TNL, Dang KT. Đánh giá tác dụng phục hồi chức năng vận động của trẻ bại nãothể co cứng bằng điện châm, thủy châm kết hợp xoa bóp bấm huyệt. http://123doc.org//document/2268573-danhgia-tac-dung-phuc-hoi-chuc-nang-van-dongcua-tre-bai-nao-the-cocung-bang-dien-cham-thuy-cham-ket-hop-xoa-bop-bam-huyet.htm

7. VUFO-NGO Resource Centre. 60,000 Vietnamese children suffer from cerebral palsy. http://www.ngocentre.org.vn/content/60000vietnamese-children-suffer-cerebral-palsy

8. Khandaker G, Smithers-Sheedy H, Islam J, et al. Bangladesh Cerebral Palsy Register (BCPR): a pilot study to develop a national cerebral palsy (CP) register with surveillance of children for CP. BMC Neurol 2015;15:173.

9. Centre for Social Assistance for Disadvantaged Children (CSADC). 2011 Annual Report -Vietnam. Ho Chi Minh, Vietnam: Christina Noble Children's Foundation, 2011. 12p.

10. Zurynski Y, Mclntyre P, Booy R, et al. Paediatric active enhanced disease surveillance: a new surveillance system for Australia. $J$ Paediatr Child Health 2013;49:588-94.

11. Marshall HS, McIntyre P, Richmond P, et al. Changes in patterns of hospitalized children with varicella and of associated varicella genotypes after introduction of varicella vaccine in Australia. Pediatr Infect Dis J 2013;32:530-7.

12. Elliott EJ, Zurynski YA, Walls T, et al. Novel inpatient surveillance in tertiary paediatric hospitals in New South Wales illustrates impact of first-wave pandemic influenza A H1N1 (2009) and informs future health service planning. J Paediatr Child Health 2012;48:235-41.

13. Khandaker G, Zurynski Y, Ridley G, et al. Clinical epidemiology and predictors of outcome in children hospitalised with influenza $A(\mathrm{H} 1 \mathrm{~N} 1)$ pdm09 in 2009: a prospective national study. Influenza Other Respir Viruses 2014;8:636-45.

14. Surveillance of Cerebral Palsy in Europe. Surveillance of cerebral palsy in Europe: a collaboration of cerebral palsy surveys and registers. Surveillance of Cerebral Palsy in Europe (SCPE). Dev Med Child Neurol 2000;42:816-24.

15. Cerebral Palsy Alliance Research Institute. Australian Cerebral Palsy 10 Register Report, 2013. http://www.cpresearch.org.au/pdfs/2013 ACPR-Report_Web.pdf (accessed on Nov 2014).

16. Bax M, Goldstein M, Rosenbaum P, et al. Executive Committee for the Definition of Cerebral Palsy. Proposed definition and classification of cerebral palsy. Dev Med Child Neurol 2005;47:571-6.

17. Rosenbaum P, Paneth N, Leviton A, et al. A report: the definition and classification of cerebral palsy April 2006. Dev Med Child Neurol Supp/ 2007;109:8-14.

18. Mutch L, Alberman E, Hagberg B, et al. Cerebral palsy epidemiology: where are we now and where are we going? Dev Med Child Neurol 1992;34:547-51.

19. Novak I, Morgan C, Adde L, et al. Early, Accurate Diagnosis and Early Intervention in Cerebral Palsy: Advances in Diagnosis and Treatment. JAMA Pediatr 2017;171:897-907.

20. Van Bang N, Van Anh NT, Van VT, et al. Surveillance of congenital rubella syndrome (CRS) in tertiary care hospitals in Hanoi, Vietnam during a rubella epidemic. Vaccine 2014;32:7065-9. 